\section{Comparative Response of Strawberries to Conidial Root-dip Inoculations and Infection by Soilborne Microsclerotia of Verticillium dahliae Kleb.}

\author{
Thomas R. Gordon \\ Plant Pathology Department, University of California, Davis, CA 95616
}

\author{
Douglas V. Shaw and Kirk D. Larson \\ Plant Sciences Department, University of California, Davis, CA 95616 \\ Additional index words. Fragaria $\times$ ananassa, conidia, microsclerotia, repeatablilty, \\ soilborne pathogen
}

\begin{abstract}
Previous studies have demonstrated significant genetic variation for susceptibility to verticillium wilt, caused by Verticillium dahliae, among strawberry (Fragaria $\times$ ananassa Duch.) genotypes adapted to California growing conditions. These evaluations have been conducted using a conidial root-dip inoculation procedure; valid application of this method in a breeding program assumes the reaction of inoculated plants will be predictive of their response to infection by more natural means. To test this expectation, we evaluated the responses of plants representing eight strawberry genotypes that were either root-dip inoculated prior to being transplanted into a fruit production field or were transplanted into soil artificially infested with pathogen propagules (microsclerotia). Both inoculation methods revealed significant variation among genotypes in all 3 years that tests were conducted and the absence of significant genotype $\times$ treatment interactions demonstrate similar rankings of genotypes with both methods. However, based on statistical repeatability, the root-dip inoculation method was more consistent over time $(R=0.759)$ than the soil inoculation method $(R=0.510)$.
\end{abstract}

Production of strawberries in California has long relied on the use of pre-plant soil fumigation with methyl bromide and chloropicrin(Wilhelm and Koch, 1956). Among the many benefits of regular fumigation has been the suppression of soilborne pathogens such as Verticillium dahliae, which causes a wilt disease of strawberries (Paulus, 1990). Although these practices have kept soil populations of the pathogen low, the fungus may still be present at both high elevation nurseries used for runner plant propagation and fruit production fields (Gordon et al., 2002). The persistence of $V$. dahliae is due to its very wide host range (Bhatt and Subbarao, 1999), its ability to survive in soil as microsclerotia (Schnathorst, 1981) and regular opportunities for introduction, as with soil or infected plant materials, into otherwise noninfested fields.

In the absence of an effective fumigant, it will be very difficult to maintain soil populations of $V$. dahliae below damaging levels (Harris and Yang, 1996). Consequently, management of verticillium wilt may require enhanced genetic resistance in commercial cultivars as a component of a disease management strategy. Previous work has demonstrated significant genetic variation for susceptibility to $V$. dahliae within germplasm in the University of California breeding program (Shaw et al., 1996) and family level selection has been shown to be an effective strategy for increasing resistance to this disease (Shaw et al., 1997).

Previous evaluations have relied on root-dip

Received for publication 9 Nov. 2004. Accepted for publication 16 Mar. 2005.
88.31-3, Cal 90.241-5, Cal 94.256-607, and Cal 92.48-1, which collectively encompass a range of susceptibilities to $V$. dahliae, were planted at the Wolfskill Experimental Orchard near Winters, Calif. ( $\left.38^{\circ} 30^{\prime} \mathrm{N}, 121^{\circ} 59^{\prime} \mathrm{W}\right)$. Runner plants representing each of these eight genotypes were subjected to one of two inoculation methods (described below), or were not inoculated (controls). The experiment, which included three replications (five runner plants/ replication) of each treatment in a randomized block design, was conducted in each of three successive years: 2000, 2001, and 2002. The procedures used were the same in each year, unless otherwise noted. This included preplant treatment of the experimental plots with 2 methyl bromide : 1 trichloronitromethane (chloropicrin) at $392 \mathrm{~kg} \cdot \mathrm{ha}^{-1}$.

The inoculation procedures were 1) rootdipping in a conidial suspension prior to planting or 2) planting into soil artificially infested with microsclerotia of $V$. dahliae. A single isolate of $V$. dahliae was used in both treatments; this isolate was originally obtained from a symptomatic strawberry plant at a strawberry runner plant nursery in California, and was demonstrated to be virulent in previous tests (Gordon et al., 2002).

Root-dip inoculations were conducted as previously described (Shaw et al., 1996), using an aqueous suspension of $1.0 \times 10^{6}$ spores $/ \mathrm{mL}$. Inoculum for soil infestation treatments was produced by inoculating sheets of sterile cellophane overlain on plates of half-strength potato dextrose agar (19.5 g Difcopotato dextrose agar plus $7.5 \mathrm{~g}$ agar/L of water) with an aqueous suspension of $10^{3}$ spores in $100 \mu \mathrm{L}$ of water. After 3 to 4 weeks of incubation at room temperature $\left(22 \pm 2^{\circ} \mathrm{C}\right)$, abundant microsclerotia formed on the cellophane. At that time, the cellophane and much of the underlying agar was placed in a blender with sufficient sterile water to allow for adequate blending. The resulting homogenate was poured into sterile sand and mixed periodically to facilitate drying. In total, 25 colonized plates were blended with sand to obtain a final volume of $10 \mathrm{~L}$.

About $0.5 \mathrm{~L}$ of soil was removed from each planting site, and soil from all planting sites (about $60 \mathrm{~L}$ ) was bulked and mixed with sand inoculum (described above). This mixture of native soil and infested sand was used to refill planting holes, into which a plant of the appropriate cultivar or breeding line was placed. The forgoing description applies to the treatments applied in 2000 and 2001. The procedure was the same in 2002, except that the volume of sand into which inoculum was incorporated was 20 rather than 10 L. Dilution rates with native soil were adjusted to achieve the same quantity of inoculum per planting site as in previous years. To estimate the inoculum density to which plant roots were exposed, a composite of eight subsamples of the infested soil was assayed using soil dilution plating as described by Gordon et al. (2002). Inoculum densities were estimated to be 415,809 , and 1419 colony forming units per gram of infested soil in 2000, 2001 and 2002, respectively.

Plants receiving root-dip inoculations were planted into the same soil as those in the soil 
Table 1. Analysis of variance results for disease ratings of plants inoculated with Vertcillium dahliae by one of two different methods in each of 3 successive years ${ }^{2}$

\begin{tabular}{lrcc}
\hline Source & df & MS & \multicolumn{1}{c}{ F } \\
\hline Year & 2 & 1.945 & 0.398 \\
Replication (year) & 6 & 0.100 & 0.892 \\
Genotype (G) & 7 & 12.71 & $13.03^{* *}$ \\
Treatment (T) & 1 & 8.434 & $33.00^{\text {*** }}$ \\
Year $\times \mathrm{G}$ & 14 & 0.836 & 1.941 \\
Year $\times \mathrm{T}$ & 2 & 4.645 & $10.79^{* *}$ \\
$\mathrm{G} \times \mathrm{T}$ & 7 & 0.570 & 1.325 \\
Year $\times \mathrm{G} \times \mathrm{T}$ & 14 & 0.431 & 1.632 \\
Error & 143 & 0.264 & \\
\hline Plant
\end{tabular}

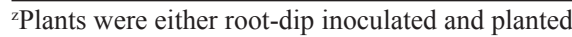
into noninfested soil, or were not root-dip inoculated but were planted into soil infested with $V$. dahliae. yPlants were inoculated either by a root-dip inoculation or by transplanting into soil infested with $V$. dahliae.

${ }^{* *}$ Significant at $P<0.01$.

Table 2. Mean resistance score $( \pm \mathrm{SE})$ for eight strawberry genotypes for each inoculation method averaged for 3 years ${ }^{\mathrm{z}}$

\begin{tabular}{lcc}
\hline & \multicolumn{2}{c}{ Inoculation method ${ }^{z}$} \\
\cline { 2 - 3 } Genotype & \multicolumn{1}{c}{ Root-dip } & Soil \\
\hline Camarosa & $2.94(0.20)$ & $3.33(0.20)$ \\
Laguna & $4.19(0.26)$ & $4.63(0.10)$ \\
Parker & $1.76(0.13)$ & $3.01(0.33)$ \\
Seascape & $3.03(0.21)$ & $3.51(0.18)$ \\
Cal 88.31-3 & $2.25(0.21)$ & $2.41(0.32)$ \\
Cal 90.241-5 & $3.65(0.31)$ & $4.16(0.14)$ \\
Cal 92.48-1 & $3.97(0.21)$ & $4.04(0.27)$ \\
Cal 94.256-607 & $4.17(0.15)$ & $4.72(0.11)$ \\
\hline
\end{tabular}

zPlants were either root-dip inoculated with a spore suspension of $V$. dahliae and planted into noninfested soil, or were not root-dip inoculated and planted into soil infested with $V$. dahliae.

inoculation treatments, but without added sand inoculum. Runner plants were root-dip inoculated and planted on a single date each year, 15, 9, and 17 Oct. for the 2000-02 trials, respectively. Two noninoculated plants of the test genotype were placed adjacent to each plot, regardless of inoculations treatments, and disease ratings were made relative to these control plants. Treatment effects were evaluated the following spring, by rating plants for symptoms of verticillium wilt on a scale of 1 to 5 , where $1=$ severely diseased and $5=$ no symptoms of disease (i.e., comparable in appearance to noninoculated controls). Individual plots were evaluated 4 to 5 times in each trial year at about 3 -week intervals beginning with the first symptoms on susceptible genotypes (16 Mar. to 10 Apr.), and a combined score was obtained as the arithmetic mean of scores for all dates (Shaw et al., 1996).

A combined mixed model analysis of variance (ANOVA) was conducted for mean disease ratings with Year and Genotype treated as random effects and Inoculation Treatment as a fixed effect using SAS procedure GLM (SAS, 1988). Further evaluation of the main effects of genotypes was conducted using simple effects analysis (Steele and Torrie, 1980) after the detection of significant year $\times$ treatment interactions. The consistency of genetic effects over years was further resolved by conducting a random effects ANOVA for each treatment separately using GLM, then obtaining estimates of variance components for each treatment using SAS procedure VARCOMP and calculating an experimental Repeatability, $\mathrm{R}$, for each inoculation method as $R=\sigma_{\mathrm{G}}{ }^{2} /\left(\sigma_{\mathrm{G}}{ }^{2}+\sigma_{\mathrm{G} \times \mathrm{Y}}{ }^{2}+\sigma_{\mathrm{E}}{ }^{2}\right)$, where $\sigma_{\mathrm{G}}{ }^{2}$ is the variance among genotypes, $\sigma_{\mathrm{G} \times \mathrm{Y}}{ }^{2}$ is the variance due to genotype $\times$ year interaction, and $\sigma_{\mathrm{E}}^{2}$ is the error variance. This statistic is equivalent to a broad-sense heritability, but is more appropriately treated as an experimental repeatability here, as the population evaluated was chosen to exhibit a range of susceptibilities, and is not a random sample of a specific reference population.

\section{Results}

Analysis of variance (ANOVA) detected highly significant main effects for both genotypes and inoculation treatments, but the year $\times$ treatment interaction effect was also significant (Table 1), complicating the interpretation of the main effects for treatments. Importantly, interaction effects involving genotypes and treatments in any combination were nonsignificant (Table 1), indicating that both inoculation methods provided similar rankings of genetic resistance. Further comparison of genotypic means calculated over all three trial years demonstrated similar infection response across inoculation treatments (Table 2). The simple correlation coefficient for these average genotypic infections scores in the two inoculation treatments was $r=$ $0.92(P<0.01)$, indicating nearly complete concordance for ratings obtained from the two evaluation methods. Analysis of simple main effects demonstrated that both root-dip and soil inoculation methods generated significant differences among genotypes in all three years ( $P<0.01$ for all comparisons), thus significant differences among genotypes occur regardless of yearly differences between infection methods. A comparison of inoculation treatment means within trial years shows that root-dip inoculations consistently produced more severe symptoms than the soil infestation treatment (Table 3), although differences were substantial only in the 2000 trial. These differences in the

Table 3. Mean disease severity ratings ${ }^{\mathrm{z}}( \pm \mathrm{SD})$ resulting from two different methods of inoculation with Vertcillium dahliae in each of 3 successive years.

\begin{tabular}{lccc}
\hline Inoculation & \multicolumn{3}{c}{ Trial year } \\
\cline { 2 - 4 } method $^{y}$ & 2000 & 2001 & 2002 \\
\hline Root-dip & $2.66 \pm 0.88$ & $3.55 \pm 1.04$ & $3.52 \pm 1.01$ \\
Soil inoculation & $3.86 \pm 0.78$ & $3.73 \pm 1.15$ & $3.60 \pm 1.01$ \\
\hline
\end{tabular}

${ }^{2}$ Plants were rated for disease on a scale of 1 to 5 , with $1=$ the most severe symptoms and $5=$ absence of symptoms.

${ }^{y}$ Root-dip $=$ plant roots immersed in a spore suspension before transplanting, soil inoculation $=$ plants not root-dipped but planted directly into soil artificially infested with microsclerotia of $V$. dahliae.

relative severity of infection over trial years likely explain the significant treatment $\times$ year interaction effects detected in the combined ANOVA (Table 1).

Estimates of the genotypic repeatability were $R=0.759$ and 0.510 for the root-dip inoculation method and the soil inoculation method, respectively (Table 4). Both genotype $\times$ year interaction and error variances were larger for the soil inoculation treatment, so the difference in precision of the two methods derives from both sources.

\section{Discussion}

The use of root-dip inoculations is predicated on the assumption that genotypes appearing resistant in these tests will display a similar phenotype when challenged with soilborne inoculum under field conditions. Our results indicate that root-dip inoculations, as conducted in our experiments, induce symptoms at least as severe as infections initiated by microsclerotia in the soil. In contrast, Maas et al. (1985) found that inoculum incorporated into the rooting medium caused greater damage to a susceptible strawberry cultivar than a root-dip inoculation. In their study, strawberries were transplanted directly into a colonized organic substrate (oat seed or vermiculite). Although colony forming units per gram were not reported, it seems likely that the effective inoculum density was substantially higher than in our study, where inoculum was diluted in sand and uninfested soil.

In the present study, both inoculation methods revealed significant differences in susceptibility among strawberry genotypes in all three years. More importantly, there was no significant interaction between inoculation method and genotype, and the correlation of genotypic mean disease ratings between the two methods was very high. This indicates that a ranking of genotypes based on susceptibility is not influenced by inoculation method and consequently the mechanism of resistance responsible for the differences detected is likely to be the same in both cases.

On the other hand, differences between treatments were not consistent over time, as indicated by the significant interaction between year and treatment (Table 1). The reasons for this interaction are not known, but most likely include environmental influences on disease development. Verticillium wilt can be strongly affected by temperature and the phenology of its host plant (Schnathorst, 1981). The impact

Table 4. Variance components and Repeatabilities for disease ratings of plants inoculated with Verticillium dahliae by two different methods ${ }^{2}$

\begin{tabular}{lcc}
\hline $\begin{array}{l}\text { Variance } \\
\text { component }\end{array}$ & $\begin{array}{c}\text { Root-dip } \\
\text { inoculation }\end{array}$ & $\begin{array}{c}\text { Soil } \\
\text { inoculation }\end{array}$ \\
\hline$\sigma_{\mathrm{G}}{ }^{2}{ }^{2}$ & 0.786 & 0.549 \\
$\sigma_{\mathrm{G} \times \mathrm{Y}}{ }^{2}$ & 0.056 & 0.189 \\
$\mathrm{\sigma}_{\mathrm{E}}{ }^{2}$ & 0.194 & 0.337 \\
$\mathrm{R}$ & 0.759 & 0.510 \\
\hline 2Plants were either root-dip inoculated with a spore
\end{tabular}

${ }^{2}$ Plants were either root-dip inoculated with a spore suspension of $V$. dahliae and planted into noninfested soil (root-dip inoculation), or were not root-dip inoculated and planted into soil infested with $V$. dahliae (soil inoculation). 
of these effects is likely to differ according to the extent of disease progression within a plant. Root-dip inoculations, which immediately expose much of the root system to infective propagules, are expected to result in systemic infections more quickly than soil inoculations, for which most infections will not occur until growing roots encounter microsclerotia in the soil. As a result, the time course of disease development may differ between plants inoculated by different methods, and this may lead to differential impacts of temperature in some years, but not others.

Although both methods gave generally similar results in all 3 years, the root-dip method provided somewhat more consistent genotypic ratings across years. This differential is documented by a measure of repeatability, based on variance components, that was $>50 \%$ higher for the root-dip assay. Greater variability in the soil inoculation method may be due, in part, to variation in inoculum densities across years, which may be attributable to differences in the extent of degradation of the cellophane on which microsclerotia were formed. This would be expected to result in greater dispersion of microsclerotia and hence the recovery of more discrete colonies on soil dilution plates. Whether or not such differences would have influenced the frequency with which growing roots encountered inoculum is unclear, but any effect on symptom development must have been small, as disease severities resulting from the soil inoculation method, averaged across all genotypes, ranged only from 3.60 to 3.86 for the three years in which the study was conducted (Table 3 ).

In conclusion, the root-dip inoculation method provides a measure of resistance to verticillium wilt that is similar to what can be discerned when plants become naturally infected by growing them in infested soil. This, coupled with its much greater ease of use, makes root-dip inoculations the method of choice in screening strawberries for resistance to verticillium wilt.

\section{Literature Cited}

Beckman, C.H. and E.M. Roberts. 1995. On the nature and genetic basis for resistance and tolerance to fungal wilt diseases of plants. Adv. Bot. Res. 21:35-77.

Bhat, R.G. and K.V. Subbarao. 1999. Host range specificity in Verticillium dahliae. Phytopathology 89:1218-1225.

Gordon, T.R., S.C. Kirkpatrick, D.V. Shaw, and K.D. Larson. 2002. Differential infection of mother and runner plant generations by Verticillium dahliae in a high elevation strawberry (Fragaria x ananassa Duch.) nursery. HortScience37:927-931.

Harris, D.C. and J.R. Yang. 1996. The relationship between the amount of Verticillium dahliae in soil and the incidence of strawberry wilt as a basis for disease risk prediction. Plant Pathol. 45:106-114.
Happstadius, I., A. Ljungberg, B. Kristiansson, and C. Dixelius. 2003. Identification of Brassica oleracea germplasm with improved resistance to verticillium wilt. Plant Breed. 122(1):30-34.

Maas, J.L., A.D. Draper, and G.J. Galletta. 1985 Inoculation methods for evaluating verticillium wilt resistance in strawberry germplasm. HortScience 20:739-741.

Paulus, A.L. 1990. Fungal diseases of strawberry. HortScience 25:885-889.

SAS Institute, Inc. 1988. SAS/STAT Users guide release 6.03, SAS Inst., Cary, N.C.

Schnathorst, W.C. 1981. Life cycle and epidemiology of Verticillium, p. 81-144. In: M.E. Mace, A.A. Bell, and C.H. Beckman (eds.). Fungal wilt diseases of plants. Academic Press, London, U.K.

Shaw, D. V., W.D. Gubler, K.D. Larson, and J. Hansen. 1996. Genetic variation for field resistance to Verticillium dahliae evaluated using genotypes and segregating progenies of California strawberries. J. Amer. Soc. Hort. Sci. 121:625-628.

Shaw, D.V., W.D. Gubler, J. Hansen, and K.D. Larson. 1997. Response to family selection for field resistance to Verticillium dahliae in California strawberries. J. Amer. Soc. Hort. Sci. 122:653-655.

Steele, R.G.D. and J.H. Torrie. 1980. Principals and procedures of statistics. 2nd ed. McGrawHill Book Co.

Wilhelm, S. and E.C. Koch. 1956. Verticillium wilt controlled. Calif. Agr. 10:3-14.

Zink, F.W., W.D. Gubler, and R.G. Grogan. 1983. Reaction of muskmelon germplasm to inoculation with Fusarium oxysporum f. sp. melonis race 2. Plant Dis. 67:1251-1255. 